\title{
Arthroscopic excision of giant cell tumor of the tendon sheath in the knee mimicking patellar tendinopathy: A case report
}

\author{
KAI GAO*, JIWU CHEN*, SHIYI CHEN and YUNXIA LI \\ Department of Sports Medicine and Arthroscopy Surgery, Huashan Hospital, Fudan University, Shanghai 200040, P.R. China
}

Received January 21, 2015; Accepted February 25, 2016

DOI: $10.3892 / 01.2016 .4419$

\begin{abstract}
Giant cell tumor of the tendon sheath (GCTTS) predominantly occurs in the tendon sheaths of the hand, but rarely in those of the knee. The current study reports the case of a 36-year-old male patient presenting with anterior knee pain. The patient was ultimately diagnosed with GCTTS in the knee mimicking patellar tendinopathy. To the best of our knowledge, this is the first case of its kind. Magnetic resonance imaging revealed a well-defined oval intra-articular lesion located at the proximal segment of the infrapatellar fat pad. The lesion was completely excised under arthroscopy and pathological examination confirmed the diagnosis of GCTTS. There was no evidence of recurrence at the 2-year follow-up examination. The findings of the present study suggest that, despite its rarity, GCTTS should be considered in the differential diagnosis of patellar tendinopathy.
\end{abstract}

\section{Introduction}

Giant cell tumor of the tendon sheath (GCTTS) predominantly occurs in the tendon sheaths of the hands (1). However, rare cases of GCTTS may occur in larger joints, such as the knee and ankle joints (1). GCTTS usually occurs in individuals aged between 30 and 50 years, with a female to male ratio of 2:1 (2). In Japan, the incidence rate of GCTTS is 1 case per 50,000 individuals (3). The most common symptom of a GCTTS is a painless swelling, and imaging studies usually reveal a well-circumscribed soft tissue mass (2). To date, only a small number of cases of GCTTS have been reported in the knee (4-11); however, to the best of our knowledge, no cases of GCTTS in the knee mimicking patellar tendinopathy have been previously reported. The most common treatment for

Correspondence to: Professor Shiyi Chen, Department of Sports Medicine and Arthroscopy Surgery, Huashan Hospital, Fudan University, 12 Wulumuqi Zhong Road, Shanghai 200040, P.R. China E-mail: cshiyi@163.com

*Contributed equally

Key words: giant cell tumor of the tendon sheath, patellar tendon, tendinopathy, knee, arthroscopy
GCTTS is local excision, which includes open surgery and arthroscopic excision (2,4-11). Previous case reports have suggested that the arthroscopic excision of GCTTS in the knee has favorable clinical outcomes $(5,11)$. The reported rate of local recurrence following surgery is $10-20 \%$ (4) and the 5-year survival rate is $96 \%$ (12). However, these recurrences can usually be controlled by surgical re-excision (2). The present study reports a case of GCTTS in the knee mimicking patellar tendinopathy, due to the clinical manifestation of anterior knee pain. The tumor was successfully treated by arthroscopic excision. Written informed consent was obtained from the patient.

\section{Case report}

In December 2012, a 36-year-old male patient presented to the Department of Sports Medicine and Arthroscopy Surgery of Huashan Hospital, Fudan University (Shanghai, China) with a history of intermittent bilateral anterior knee pain for 9 months. The pain was stronger in the right knee compared with the left, and it typically appeared following extensive walking or during stair climbing, but disappeared at rest and during the night. The patient often cycled but denied any history of knee trauma. Physical examination revealed tenderness at the insertion of the patellar tendon and proximal patellar tendon in both knees. No effusion was observed and no mass was palpable. The range of motion in both knees was normal $\left(0-140^{\circ}\right)$. Plain radiographs of both knees were normal. Magnetic resonance imaging (MRI; Signa 1.5 T; GE Healthcare, Milwaukee, WI, USA) of the right knee detected an oval lesion located at the proximal segment of the infrapatellar fat pad, with low signal intensity on T1-weighted images and high signal intensity on T2-weighted images (Fig. 1). MRI of the left knee revealed no abnormal findings and all laboratory tests were normal. Thus, a diagnosis of GCTTS of the right knee was determined.

Under spinal anesthesia (10 mg bupivacaine hydrochloride; Shanghai Harvest Pharmaceutical Co., Ltd., Shanghai, China), which was administered for 75 min, right knee arthroscopy was performed using the superolateral and anterolateral approaches, as follows: The arthroscope was introduced through the superolateral portal, followed by the insertion of a $4.5 \mathrm{~mm}$ motorized shaver (Smith \& Nephew Endoscopy, Andover, MA, USA) through the anterolateral portal. Following removal of the synovium using a motorized shaver, a $2.0 \times 1.5 \times 1.2-\mathrm{cm}$ well-defined yellow mass covered by synovium was observed 
A

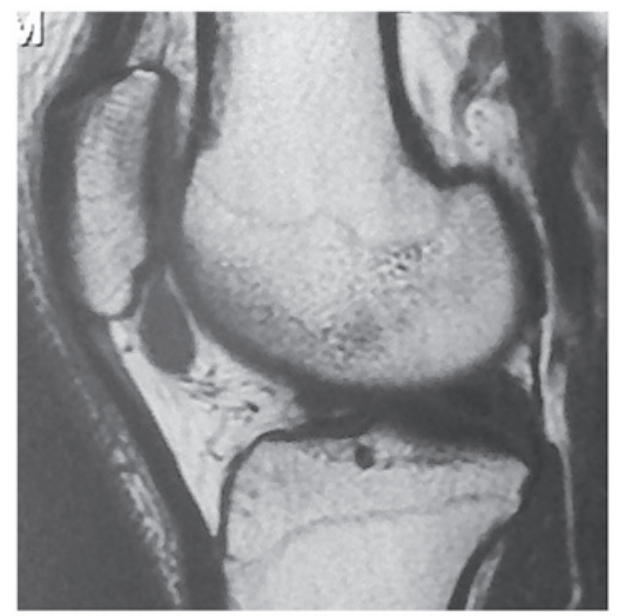

B

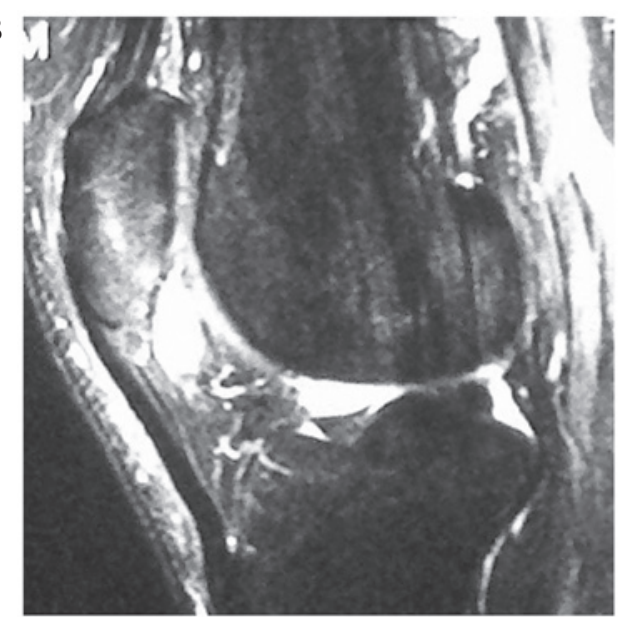

Figure 1. (A) Low signal intensity T1-weighted and (B) high signal intensity T2-weighted sagittal magnetic resonance imaging scans revealing an oval giant-cell tumor of the tendon sheath located at the proximal segment of the infrapatellar fat pad.

laterally adjacent to the distal patella (Fig. 2A). The tumor was removed using forceps (Shanghai Medical Instruments Co., Ltd., Shanghai, China) via the anterolateral approach, and the motorized shaver and a radiofrequency ablation device $(3.75 \mathrm{~mm}$ radiofrequency ablation wand; ArthroCare Corporation, Austin, TX, USA) were utilized to remove the peripheral synovium of the tumor, revealing the fat underneath the tumor (Fig. 2B). The pain was relieved immediately following surgery. The resected tissue was fixed in 10\% formalin (Shanghai Ling Feng Chemical Reagent Co., Ltd., Shanghai, China), paraffin (Leica Biosystems Nussloch GmbH, Nussloch, Germany)-embedded and cut into 5- $\mu \mathrm{m}$ sections using a microtome (Leica RM2235; Leica Biosystems Nussloch $\mathrm{GmbH}$ ), prior to staining with hematoxylin and eosin (Baso Diagnostics Inc., Zhuhai, China). Staining was visualized under a microscope (Eclipse 55i; Nikon Corporation, Tokyo, Japan). Pathological examination of the lesion revealed closely packed medium-sized mononuclear cells with a variable admixture of giant cells containing fat and hemosiderin, and evident stromal fibrosis and hyalinosis. The mononuclear component comprised two types of cells: small histiocyte-like cells, which represent the main cellular component, and larger cells. A diagnosis of GCTTS was subsequently confirmed by pathological examination (Fig. 2C), according to the World Health Organization Classification of tumors of
A

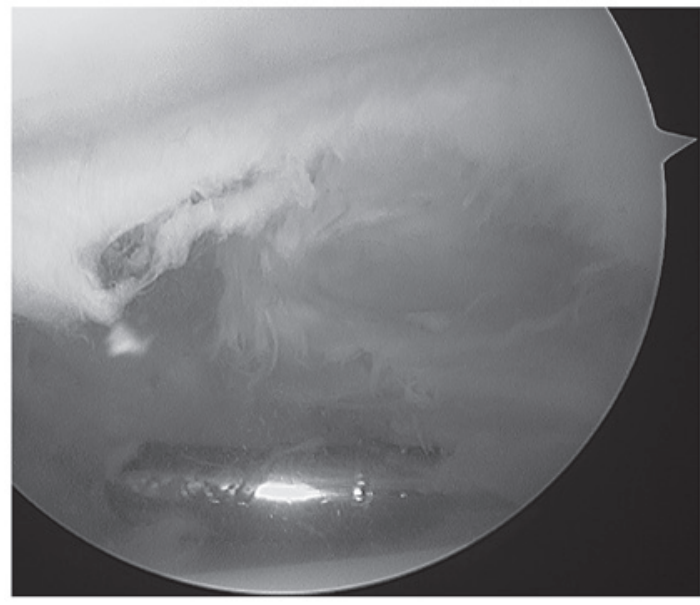

B

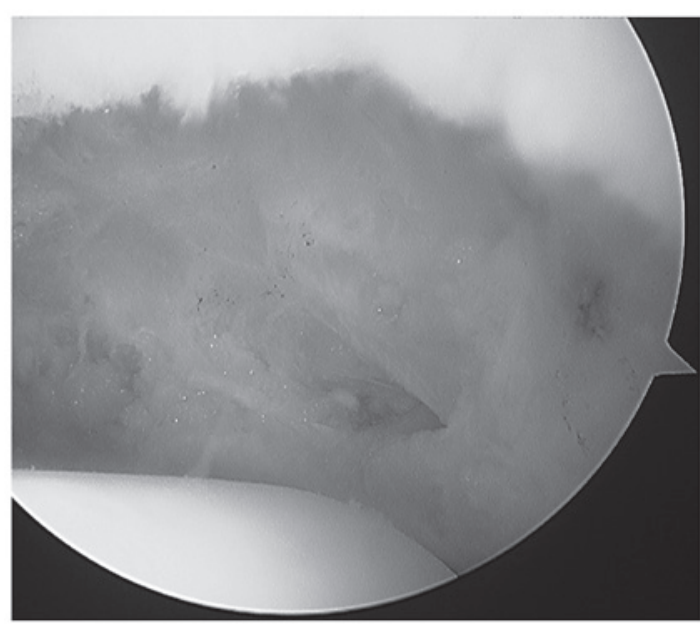

C

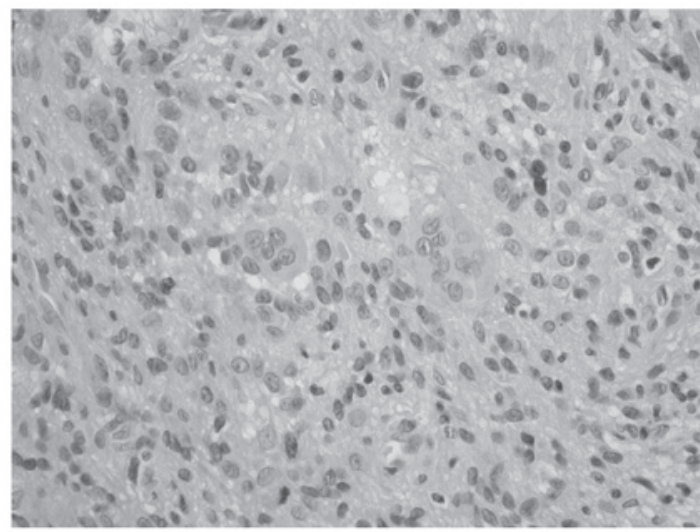

Figure 2. (A) Preoperative arthroscopic image of the right knee showing that the giant cell tumor of the tendon sheath (GCTTS) was laterally adjacent to the distal patellar. (B) Postoperative arthroscopic image of the right knee, following complete removal of the tumor. (C) Histology of the GCTTS (hematoxylin and eosin staining; original magnification, $\mathrm{x} 400$ ).

the soft tissue and bone (2). No postoperative complications occurred and the patient returned to full activity 6 weeks after surgery. Furthermore, there was no evidence of recurrence at the 2-year follow-up examination.

\section{Discussion}

Anterior knee pain is a common symptom in orthopaedic practice. Patellar tendinopathy is considered to be a common cause 
of anterior knee pain, while the presence of tumors is a particularly rare cause $(9,13)$. The clinical presentation of GCTTS in the knee is typically non-specific, thus, its clinical diagnosis is challenging (4-11). In the present study, the GCTTS in the knee mimicking patellar tendinopathy was located just posterior to the proximal patellar tendon. Therefore, it was considered that the GCTTS originated from the patellar tendon, as they were in close proximity to one another. Thus, the present study suggests that GCTTS should be considered in the differential diagnosis of patellar tendinopathy. In such cases, physical examination is insufficient to make a definitive diagnosis of the tumor. MRI scans are required to establish the diagnosis of GCTTS (14); GCTTSs appear as well-circumscribed lesions with a low signal intensity on T1-weighted images and a high signal intensity on T2-weighted images (11).

Surgical treatment options for GCTTS in the knee include arthroscopic excision and open surgery. Arthroscopy has been applied in the diagnosis and treatment of this type of tumor $(5,11)$. The advantage of arthroscopic excision is that it is minimally invasive. Furthermore, during arthroscopy, accompanied disorders can be simultaneously diagnosed and treated (5). However, GCTTSs most commonly arise from patellar tendons and anterior cruciate ligaments (4-11). If the GCTTS is covered by synovium, as was the case with the present patient, direct arthroscopic visualization of the tumor is not possible. In such cases, a motorized shaver should be used to remove the synovium, thereby exposing the underlying tumor. Open surgery allows adequate local excision of tumors invading the surrounding tissue and thus, exhibits a decreased risk of local recurrence when compared with arthroscopy (4).

In conclusion, despite its rarity, GCTTS should be considered in the differential diagnosis of patellar tendinopathy. Furthermore, MRI is essential for the preoperative diagnosis of this type of tumor. Regarding treatment options, the arthroscopic excision of GCTTs of the tendon sheath in the knee can have favorable outcomes.

\section{References}

1. Ushijima M, Hashimoto H, Tsuneyoshi M and Enjoji M: Giant cell tumor of the tendon sheath (nodular tenosynovitis). A study of 207 cases to compare the large joint group with the common digit group. Cancer 57: 875-884, 1986.

2. de St. Aubain Somerhausen N and Dal Cin N: Giant cell tumour of tendon sheath. In: World Health Organization Classification of Tumors. Pathology and Genetics of Tumors of Soft Tissue and Bone. Fletcher CDM, Krishnan Unni K and Mertens F (eds). IARC Press, Lyon, pp110-111, 2002.

3. Monaghan H, Salter DM and Al-Nafussi A: Giant cell tumour of tendon sheath (localised nodular tenosynovitis): Clinicopathological features of 71 cases. J Clin Pathol 54: 404-407, 2001

4. Akahane T, Mori N and Yoshida K: Giant cell tumor of the tendon sheath extending around the patellar tendon and invading the knee joint and tibia: A case report. Oncol Lett 8: 2800-2802, 2014.

5. Cho HJ, Lee SH, Han SB, Lee DK, Kim CH and Lee DH: Bilateral tenosynovial giant cell tumor of the knee accompanied by chronic ACL tear. J Orthop Sci 17: 93-97, 2012.

6. Chechik O, Amar E, Khashan M and Morag G: Giant cell tumors in the patellar tendon area. J Knee Surg 23: 115-119, 2010.

7. Sun C, Sheng W, Yu H and Han J: Giant cell tumor of the tendon sheath: A rare case in the left knee of a 15-year-old boy. Oncol Lett 3: 718-720, 2012.

8. Lüthje P and Nurmi-Lüthje I: Tenosynovial juxta-articular giant-cell tumour of the knee-an unusual location of the tumour. Acta Orthop Belg 72: 772-774, 2006.

9. Relwani J, Factor D, Khan F and Dutta A: Giant cell tumour of the patellar tendon sheath-an unusual cause of anterior knee pain: A case report. Knee 10: 145-148, 2003.

10. Abdullah A, Abdullah S, Haflah NH and Ibrahim S: Giant cell tumor of the tendon sheath in the knee of an 11-year-old girl. J Chin Med Assoc 73: 47-51, 2010.

11. Carls J, Kohn D and Maschek H: Benign giant-cell tumor of the patellar ligament. Arthroscopy 14: 94-98, 1998.

12. Somerhausen NS and Fletcher CD: Diffuse-type giant cell tumor: Clinicopathologic and immunohistochemical analysis of 50 cases with extraarticular disease. Am J Surg Pathol 24: 479-492, 2000.

13. Kodali P, Islam A and Andrish J: Anterior knee pain in the young athlete: Diagnosis and treatment. Sports Med Arthrosc 19: 27-33, 2011.

14. Jelinek JS, Kransdorf MJ, Shmookler BM, Aboulafia AA and Malawer MM: Giant cell tumor of the tendon sheath: MR findings in nine cases. AJR Am J Roentgenol 162: 919-922, 1994. 